\title{
Efficacy and Safety of Nadifloxacin for Bacterial Skin Infections: Results from Clinical and Post-Marketing Studies
}

Varsha Narayanan $\cdot$ Salman Motlekar $\cdot$ Ganesh Kadhe $\cdot$ Seema Bhagat

To view enhanced content go to www.dermtherapy-open.com

Received: July 7, 2014 / Published online: September 12, 2014

(c) The Author(s) 2014. This article is published with open access at Springerlink.com

\section{ABSTRACT}

Introduction: Skin and soft tissue infections involve microbial invasion of the skin and underlying soft tissues and are estimated to affect $7-10 \%$ of hospitalized patients worldwide. Nadifloxacin, a topical fluoroquinolone, has been shown to be effective against aerobic Gram-negative, Grampositive (including MRSA and coagulasenegative staphylococci), and anaerobic bacteria. However, there is paucity of data comparing efficacy and safety of $1 \%$ nadifloxacin with other anti-bacterials for skin infections in Indian patients.

Methods: This article presents the results of one post-marketing surveillance (PMS) and three randomized, open, non-blinded, multi-centric clinical studies that compared nadifloxacin with mupirocin and framycetin, and nadifloxacin

Electronic supplementary material The online version of this article (doi:10.1007/s13555-014-0062-1) contains supplementary material, which is available to authorized users.

V. Narayanan · S. Motlekar $(\bowtie) \cdot$ G. Kadhe .

S. Bhagat

Wockhardt Ltd., Wockhardt Towers, Bandra Kurla

Complex, Mumbai 400051, India

e-mail: SMotlekar@wockhardt.com with fusidic acid. Patients in India, aged from 1 to 65 years old, suffering from mild to moderate bacterial skin infections including impetigo, secondarily infected wounds, folliculitis, infected atopic dermatitis, and furunculosis were randomly allocated to three treatment groups within the studies. Efficacy was assessed by the evaluation of symptoms of erythema, exudation, swelling, pruritus, crusting, pain and tenderness in all the studies.

Results: A total of 272 subjects were enrolled in the study and subjects were randomly assigned to one of the three treatment groups; 92 in the nadifloxacin group, 90 in the mupirocin group, and 90 in the framycetin group. A significant reduction in the mean scores for bacterial infection symptoms in the nadifloxacin groups was observed when compared to mupirocin, framycetin and fusidic acid groups. Both physician and patients rated nadifloxacin as excellent (complete remission of symptoms) on a 4-point scale in the studies. No adverse events (AEs) were reported in the clinical studies. In the PMS, only two patients (of 329, 0.6\%) reported AEs including burning and itching, one in each patient that had resolved at the time of reporting. 
Conclusion: Nadifloxacin, a fluoroquinolone, is a new alternative topical agent in the treatment of bacterial skin infection with minimal AEs.

Keywords: Bacterial skin infections; Dermatology; Fluoroquinolone; Nadifloxacin; SSTIs; Topical antibiotic

\section{INTRODUCTION}

Skin and soft tissue infections (SSTIs) involve microbial invasion of the skin and underlying soft tissues and are estimated to affect $7-10 \%$ of hospitalized patients worldwide. The estimated incidence rate of SSTIs is 24.6 per 1,000 personyears [1]. A study from India reported that the prevalence of infections caused by methicillinresistant Staphylococcus aureus (MRSA) was 42\% in 2008 and $40 \%$ in 2009 [2]. Common superficial bacterial infections of the skin include impetigo, folliculitis, furunculosis, cellulitis, acne and others. Topical antibiotics like mupirocin and fusidic acid are the common topical anti-bacterials used for these bacterial skin infections [3-5].

Nadifloxacin, a topical fluoroquinolone, acts by inhibiting the configuration of negative supercoiling of bacterial DNA, catalyzed by DNA gyrase. DNA gyrase is an enzyme present in every bacterium and is essential for DNA replication, transcription, and recombination [6]. Nadifloxacin has been shown to be effective against aerobic Gram-negative, Gram-positive (including MRSA and coagulase-negative staphylococci), and anaerobic bacteria [7-10]. It is approved for use in acne treatment and skin infections in Japan [11].

Nadifloxacin has shown good safety and efficacy against several bacteria. Previous in vitro studies for bacterial skin infections found nadifloxacin to be highly potent against aerobic and anaerobic bacteria such as Propionibacterium species, Streptococcus species, and Staphylococcus species [10, 12, 13]. Nadifloxacin has been in use for treatment of acne vulgaris for the past two decades and has shown good safety and efficacy profiles $[11,14-$ 16]. However, nadifloxacin has not been frequently used to treat bacterial infections of the skin.

A phase II trial of nadifloxacin $1 \%$ cream in 101 patients with impetigo, secondarily infected wounds, folliculitis, sycosis vulgaris, and impetiginized dermatitis reported a significant reduction in the degree of erythema, exudation, swelling, pain, pruritus, erosion, crusts and scaling, and eradication of causative bacteria. The adverse events (AEs) reported were infrequent with only three patients complaining of erythema, itching, and inflammatory swelling on day 4 and day 7 of the study. No serious AEs were reported $[17,18]$.

There is a paucity of data comparing efficacy and safety of $1 \%$ nadifloxacin with other antibacterials for skin infections in Indian patients. This article presents the results of four studies ( 3 randomized controlled trials and 1 postmarketing surveillance) conducted on an Indian population to investigate the efficacy and safety of nadifloxacin Nadoxin $^{\mathrm{TM}}$, Wockhardt Ltd., Mumbai, India) in the treatment of mild to moderate bacterial skin infections when compared with other antibacterials including mupirocin, framycetin, and fusidic acid.

\section{METHODS}

\section{Study Population}

Indian subjects, aged 1-65 years old, suffering from mild to moderate bacterial skin infections 
that included impetigo, secondarily infected wounds, folliculitis, infected atopic dermatitis, furunculosis and those willing to sign informed consent were enrolled in the study. Patients with a history of hypersensitivity to aminoglycosides, quinolones, and mupirocin, patients on topical anti-bacterial treatment during 1 week prior to study and those suffering from any other severe bacterial skin infections were not enrolled in the study. Patients with a history of chronic alcohol/drug abuse, those on another investigational drug during the previous 12 weeks, presence of severe concomitant diseases, patients receiving any systemic antibiotics during 2 weeks prior to the study and pregnant women or nursing mothers were excluded from the study.

\section{Compliance with Ethics Guidelines}

All procedures followed were in accordance with the ethical standards of the responsible committee on human experimentation (institutional and national) and with the Helsinki Declaration of 1975, as revised in 2000 and 2008. Informed consent was obtained from all patients for being included in the studies.

\section{Study Design}

The three clinical studies were comparative, open, multi-centric, and randomized with study duration of 14 days and the other was a phase IV study. Eligible subjects in study 1 were randomly allocated to either of the three treatment groups; the nadifloxacin group, the mupirocin group, or the framycetin group. Subjects in study 2 and study 3 were randomly allocated to receive either nadifloxacin or fusidic acid (Fig. 1). All the subjects were followed up at day 3, day 7 and day 14 .

The phase IV study was an observational, open-label, non-comparative, multi-centric, post-marketing surveillance (PMS) conducted across 125 centers in India. Duration for the study was 7 days and extended to 14 days for those subjects who did not show any improvement in the initial 7 days.

\section{Assessment}

The primary outcome variables in all the three studies were improvement in scores (on a scale of 0 -absent to 3-severe) of erythema, crusting, exudation, swelling, pruritus, pain and tenderness. In study 1 , the number of lesions and size of wound scores were also assessed.

The secondary outcome variable included global assessment by the patient and the investigator on a 4-point scale (Excellentcomplete remission, Good-acceptable remission, Fair-slight/incomplete remission, and Poor-unchanged/aggravated). Safety/ tolerability was assessed based on AEs experienced by the patients during the treatment period.

\section{Dosage Schedule}

In study $1,1 \%$ nadifloxacin cream, 2\% mupirocin or $1 \%$ framycetin ointment were given twice daily, once in the morning and once in the evening for at least 7 days and maximum up to 14 days.

In study 2 and study 3, 1\% nadifloxacin or $2 \%$ fusidic acid were given twice daily, once in the morning and once in the evening for at least 7 days and maximum up to 14 days.

In the PMS study, $1 \%$ nadifloxacin cream was used to treat enrolled patients, and the dosage schedule varied from patient to patient. Majority $(n-101)$ of the patients received the study drug once in the morning and once in the evening for a period of 1 week. 


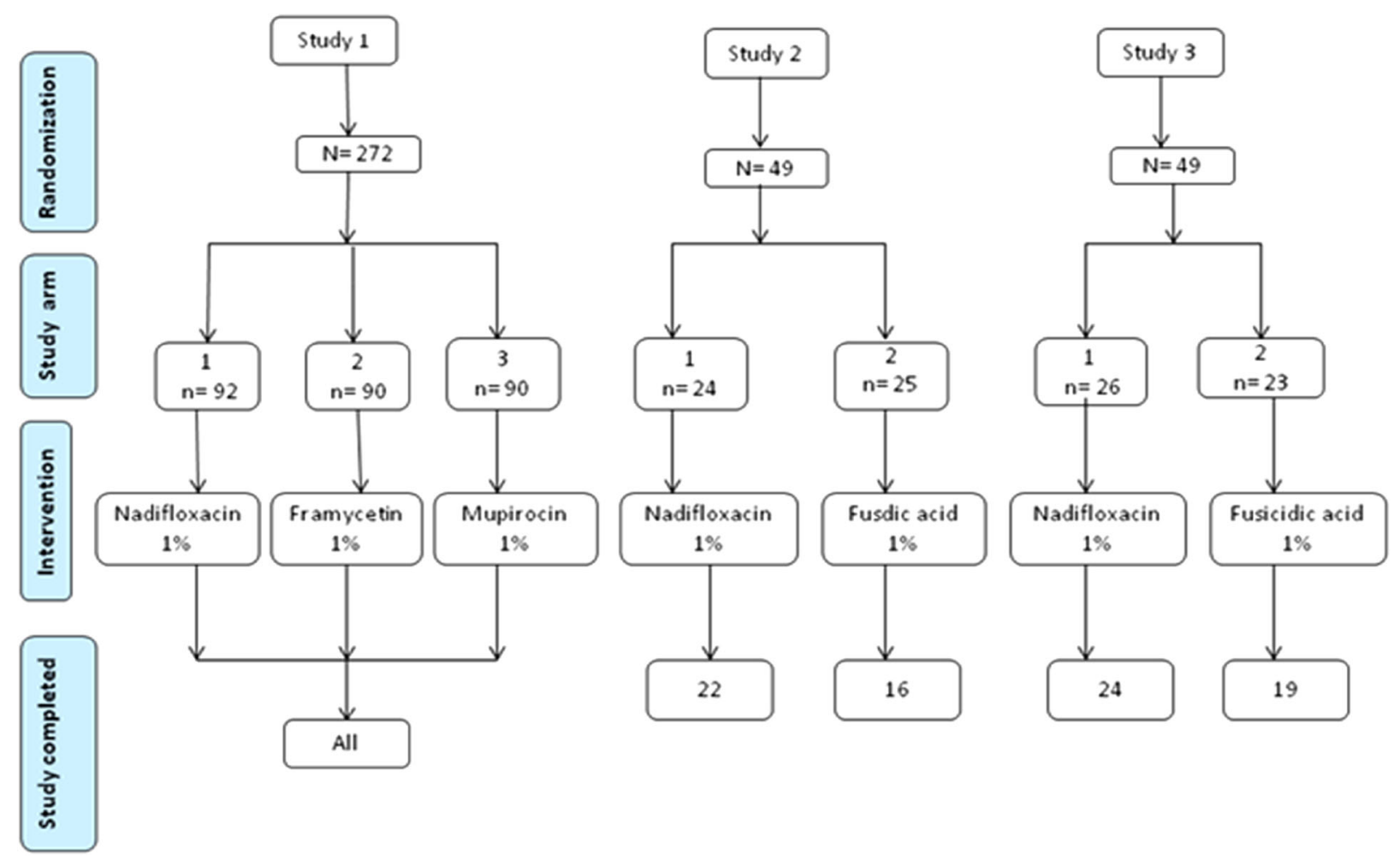

Fig. 1 Study design and patient disposition

\section{Statistical Analysis}

All the parameters were measured on a continuous scale, expressed as mean $\pm \mathrm{SD}$ and compared using Student's $t$ test. All the parameters were subjected to Chi-Squared test. Wilcoxon signed-rank and Chi-squared tests were used to analyze the categorical data.

\section{RESULTS}

\section{Subjects}

\section{Study 1}

A total of 272 subjects were enrolled in the study and all of them completed the study. Subjects were randomly assigned to one of three treatment groups; 92 in the nadifloxacin group, 90 in the mupirocin group, and 90 in the framycetin group. The demographic details of the patients are presented in Table 1. Only two patients from nadifloxacin group had a previous history of other disease, whereas none of the patients from mupirocin group and framycetin group had any disease history. History of previous drug intake was reported in 33 (35.9\%) patients from nadifloxacin group, 37 (41.1\%) from mupirocin group and 37 (41.1\%) from framycetin group.

\section{Study 2}

This study enrolled 49 patients, aged 1-65 years old, of which 38 patients (nadifloxacin group22, fusidic acid group-16) completed the study. The patient's profiles in both the groups were broadly similar (Table 1) with a majority of them suffering from impetigo, pyoderma or boils, periporitis and miscellaneous secondary infections. 


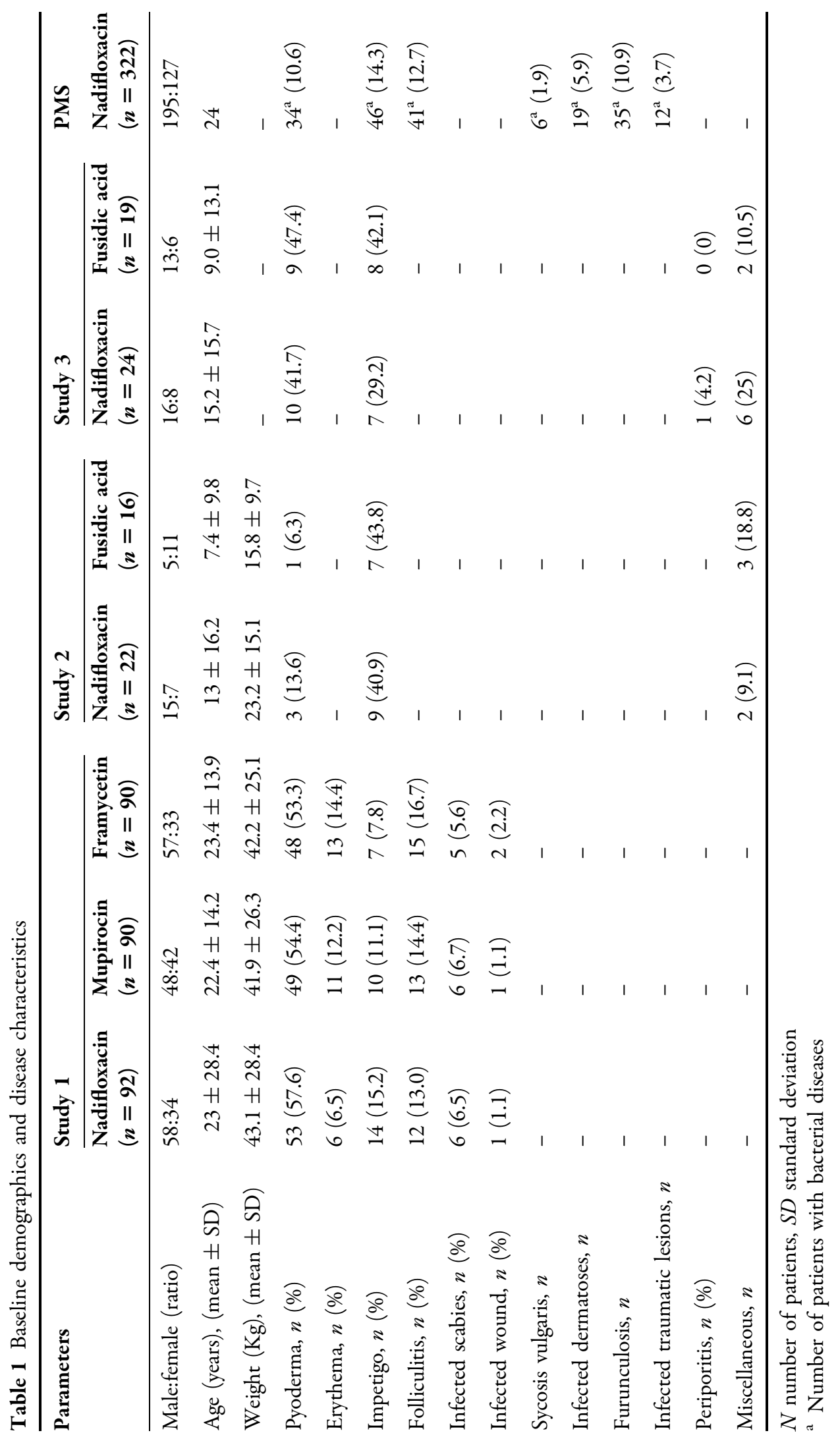




\section{Study 3}

Of 49 patients (1-60 years old) enrolled in the study, 43 patients (nadifloxacin group-24, fusidic acid group-19) completed the study. The majority of the patients were men (nadifloxacin group-67\%, fusidic acid group-68\%). The mean age of the patients in the nadifloxacin group and the fusidic acid group was $15.2 \pm 15.7$ years and $9.0 \pm 13.1$ years, respectively (Table 1 ).

\section{Phase IV Study}

Of the 329 patients enrolled in 125 centers across India, 323 patients completed the study. The majority of the patients were men (61\%), $317(98.4 \%)$ patients were aged 60 years or less and $6(1.9 \%)$ were more than 60 years old with a mean age of 24 years (range 3 months80 years).

\section{ASSESSMENT OF EFFICACY}

\section{Study 1}

\section{Primary Parameters}

The clinical cure rates were significantly higher in the nadifloxacin and mupirocin groups on day 3 (70.7\% and $72.2 \%$, respectively) and day 7 (97.8\% and $97.8 \%$, respectively) as compared with framycetin group $(46.6 \%$ on day 3 and $0.8 \%$ on day $7 ; p<0.05$ ) (Fig. 2). A significant reduction was reported in the mean scores for pain and tenderness on days 3, 7 and 14 as compared with baseline scores in all the three treatment groups $(p<0.05)$. The reduction in mean score was similar in the nadifloxacin and mupirocin groups and was significantly greater than that in the framycetin group $(p<0.05)$. Similar results were found for other parameters like the number of lesions, size of lesions, erythema, crusting, exudation, swelling and pruritus. Figure $3 a$ depicts the improvement in clinical parameters with nadifloxacin and mupirocin.

\section{Secondary Parameters}

Global Assessment of the Treatment by Patients Overall, 44 (47.8\%) patients rated nadifloxacin as excellent (complete remission of symptoms) compared to only 11 (12.2\%) from the mupirocin group and 7 (7.8\%) from the framycetin group, whereas $46(50 \%)$ from the nadifloxacin group and 58 (64.4\%) from the mupirocin group rated the therapy as good (acceptable remission of symptoms). Eight (8.9\%) patients from the framycetin group rated it as poor (unchanged/aggravated) compared to only 3 (3.3\%) from the mupirocin group and zero patients from the nadifloxacin group (Fig. 4a).

\section{Global Assessment of the Treatment by} Physician In 43 (46.7\%) patients, the investigator rated nadifloxacin as excellent (complete remission of symptoms), whereas in only $19(11.1 \%)$ patients from the mupirocin group and $9(10 \%)$ patients from the framycetin group, the investigator rated nadifloxacin as excellent (complete remission of symptoms). In 44 patients $(47.8 \%)$ from the nadifloxacin group and 56 (62.2\%) from the mupirocin group, the investigator rated the therapy as good (acceptable remission of symptoms). In 14 patients (15.6\%) from the framycetin group, the investigator rated it as poor (unchanged/ aggravated) against zero patients from the nadifloxacin and mupirocin groups (Fig. $4 \mathrm{~b}$ ).

\section{Study 2}

\section{Primary Parameters}

The mean severity scores for almost all the signs/symptoms like erythema, exudation, 

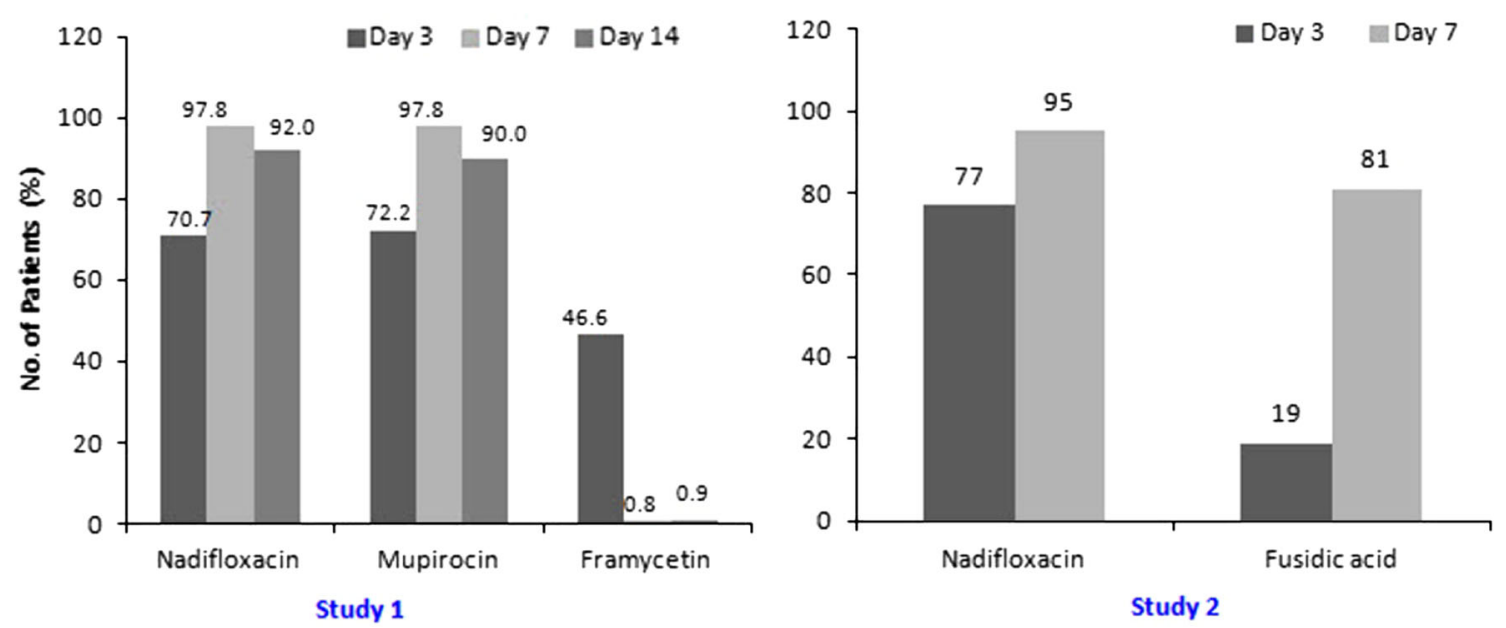

Fig. 2 Clinical cure rate (\%) in study 1 and study 2

swelling, pruritus, pain and tenderness were significantly reduced on day 3 and day 7 , compared with the baseline in both the treatment groups $(p<0.05)$. On day 3 , the mean severity scores for signs/symptoms like crusting, exudation, swelling and pruritus were significantly reduced in the nadifloxacin group when compared to the fusidic acid group $(p<0.05)$. The majority of the evaluable symptoms were relieved in more patients in the nadifloxacin group on day 3 compared to the number of patients in the fusidic acid group (Fig. 3b). However, the majority of the evaluable symptoms were relieved on day 7 in both the treatment groups. The lesions were healed in $77 \%$ and $95 \%$ of patients on day 3 and day 7 , respectively, in the nadifloxacin group compared to $19 \%$ and $81 \%$ of patients in the fusidic acid group (Fig. 2).

\section{Secondary Parameters}

Global Assessment by Patients All 22 (100\%) patients in the nadifloxacin group rated the overall response to the application of the study cream as excellent (complete remission of symptoms) compared to only 6 patients (37.5\%) from the fusidic acid group. The remaining $10(62.5 \%)$ patients in the fusidic acid group rated the therapy as good (acceptable remission of symptoms) due to inadequate response on day 3 (Fig. 4a).

Global Assessment by Physician The physician rated the overall response to the application of the study cream as excellent (complete remission of symptoms) in all 22 patients (100\%) in the nadifloxacin group compared to 4 patients (25\%) from the fusidic acid group. The physicians rated the overall response to fusidic acid as good (acceptable remission of symptoms) and fair (slight/ incomplete remission of symptoms) in 5 (31.3\%) and 7 (43.8\%) patients, respectively, due to an inadequate response and persistence of the clinical symptoms on day 3 (Fig. 4b).

\section{Study 3}

\section{Primary Parameters}

The mean severity scores for almost all the signs and symptoms like erythema, exudation, swelling, pruritus, pain and tenderness were significantly reduced on day 3 , day 7 and day 14 (except tenderness in the fusidic acid group) in 
both groups. At day 7, the mean severity score for pruritus was significantly reduced in the nadifloxacin group and the fusidic acid group $(p<0.05)$. There was no statistically significant difference for mean severity scores for other symptoms and signs between two groups (Fig. 3c). The lesions were healed in $42 \%$ and $83 \%$ of patients on day 3 and day 7 , respectively, in the nadifloxacin group compared to $21 \%$ and $63 \%$ of patients in the a 2

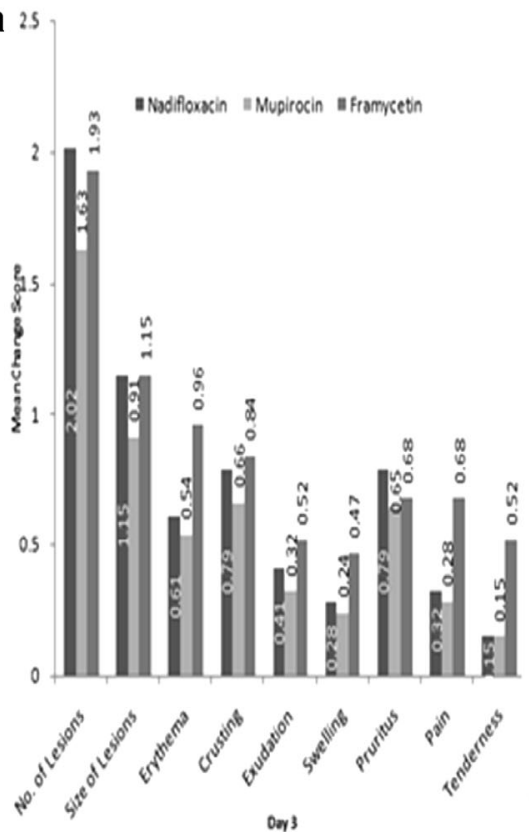

Girical Evaluation Parameters

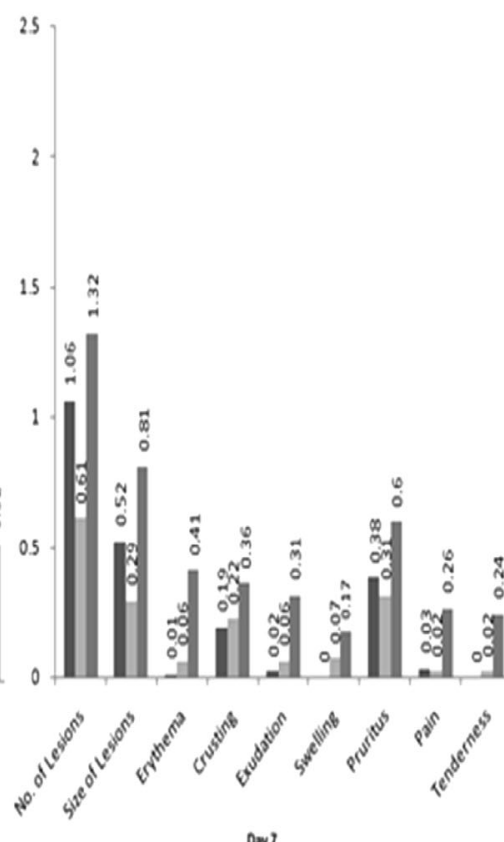

0xy
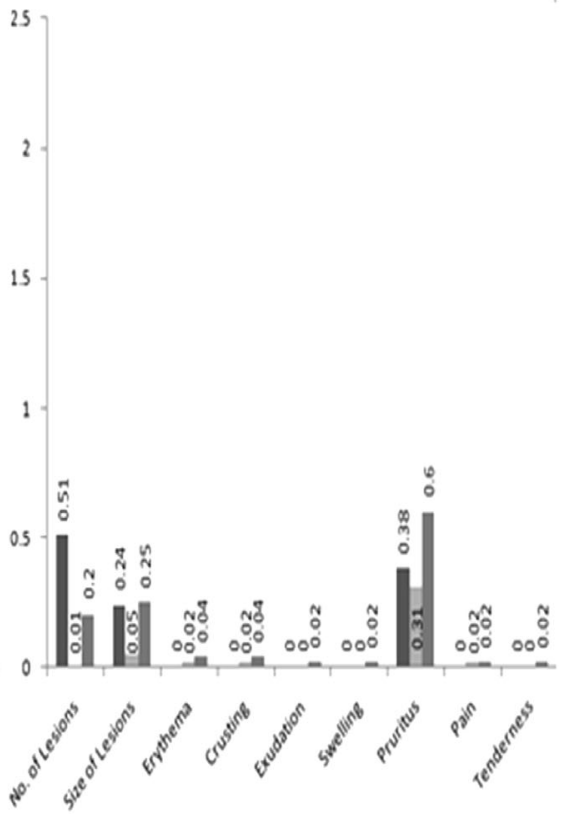

Ory 14

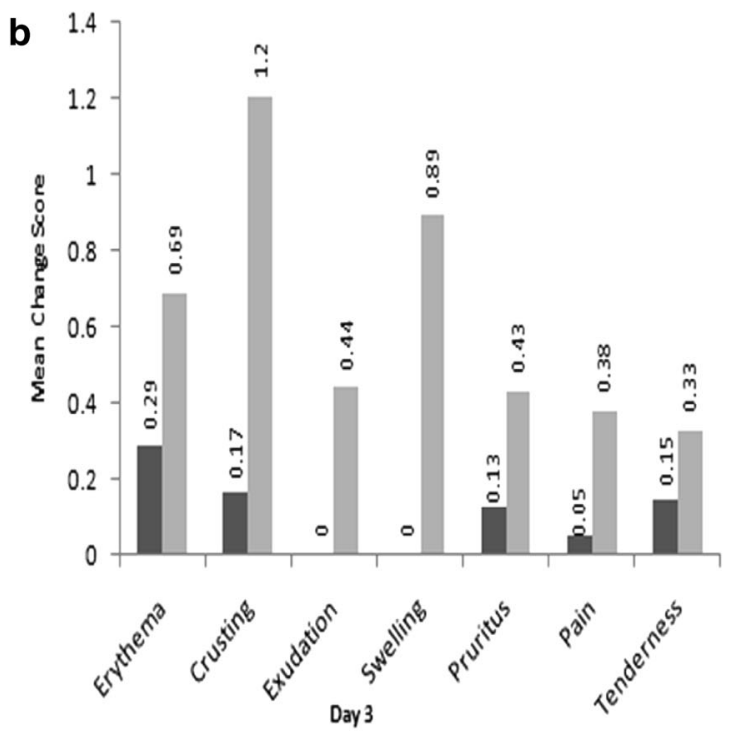

Clinical Evaluation Parameters

Fig. 3 a Improvement in mean score for evaluable symptoms at day 3 , day 7 and day 14 in study 1 . b Improvement in mean score for evaluable symptoms at day 3 and day 7 in

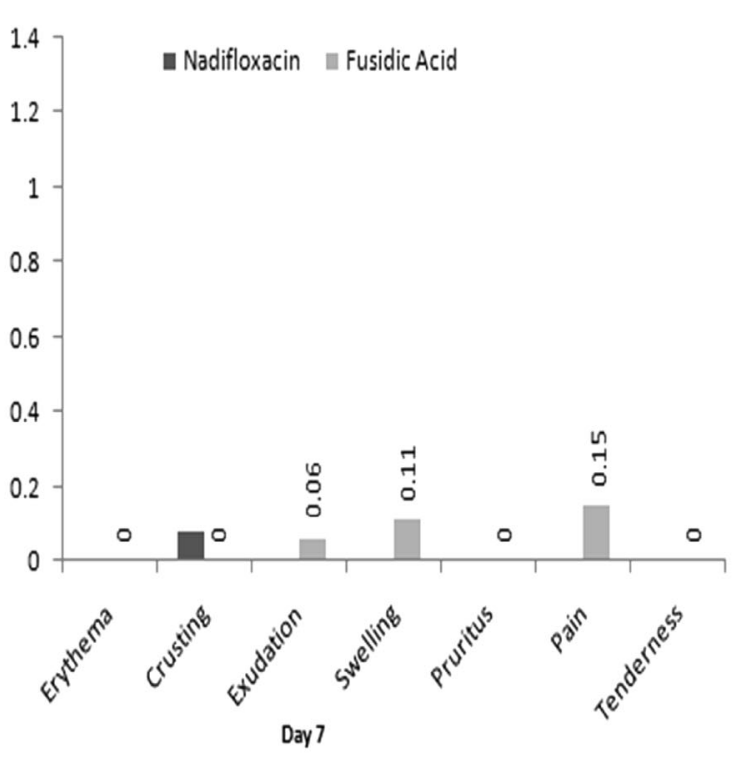

study 2. c Improvement in mean score for evaluable symptoms at day 3 , day 7 and day 14 in study 3 

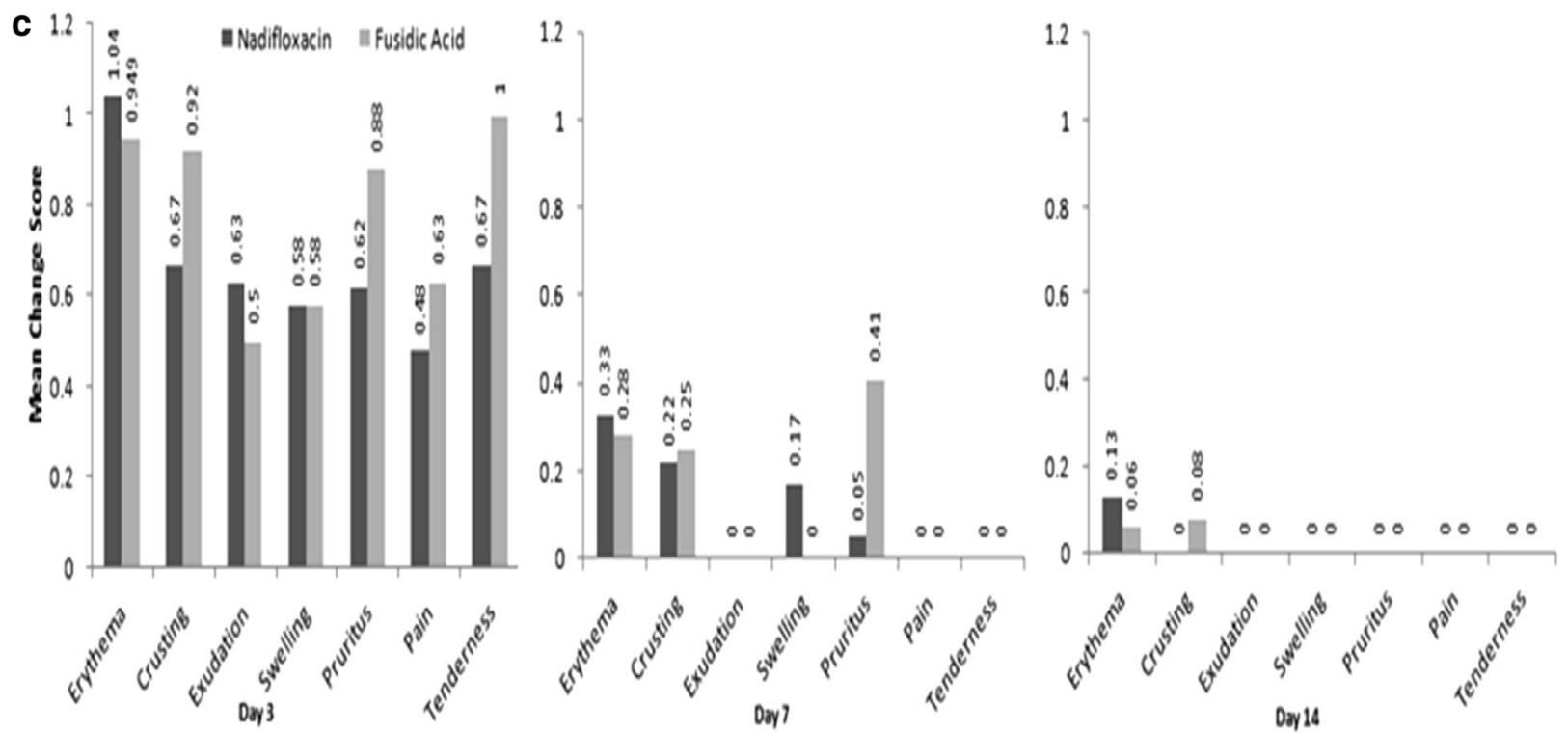

Clinical Evaluation Parameters

Fig. 3 continued

fusidic acid group. Thus, nadifloxacin resulted in healing of bacterial skin infection lesions in a higher number of patients when compared to the fusidic acid group.

\section{Secondary Parameters}

Global Assessment by Patient In the nadifloxacin group, 11 (45.8\%) patients rated the overall response to the application of the study cream as excellent (complete remission of symptoms) compared to only $3(15.8 \%)$ patients from the fusidic acid group. The remaining 13 (54.2\%) patients from the nadifloxacin group rated the therapy as good (acceptable remission of symptoms), whereas $12(63.2 \%)$ and $4(21.1 \%)$ patients from the fusidic acid group rated the therapy as good (acceptable remission of symptoms) and fair (slight/incomplete remission of symptoms), respectively (Fig. 4a).

Global Assessment by Investigator The investigator rated the overall response to the application of the study cream on a 4-point scale as excellent (complete remission of symptoms) in $11(45.8 \%)$ patients in the nadifloxacin group compared to 4 (20.5\%) patients) in the fusidic acid group. The remaining $12(50 \%)$ and $1(4.2 \%)$ patients from the nadifloxacin group received good (acceptable remission of symptoms) and fair (slight/incomplete remission of symptoms) response ratings from the investigator, compared with 11 (57.9\%) and 4 (21.1\%) patients from fusidic acid group, respectively (Fig. 4b).

\section{Phase IV}

\section{Primary Parameters}

Clinical efficacy of nadifloxacin was evaluated by assessing the improvement in primary parameters including clinical experience of the lesions in term of erythema, exudation, swelling, pruritus, crusting, pain, tenderness before study, at day 7 and day 14 (Table 2). 

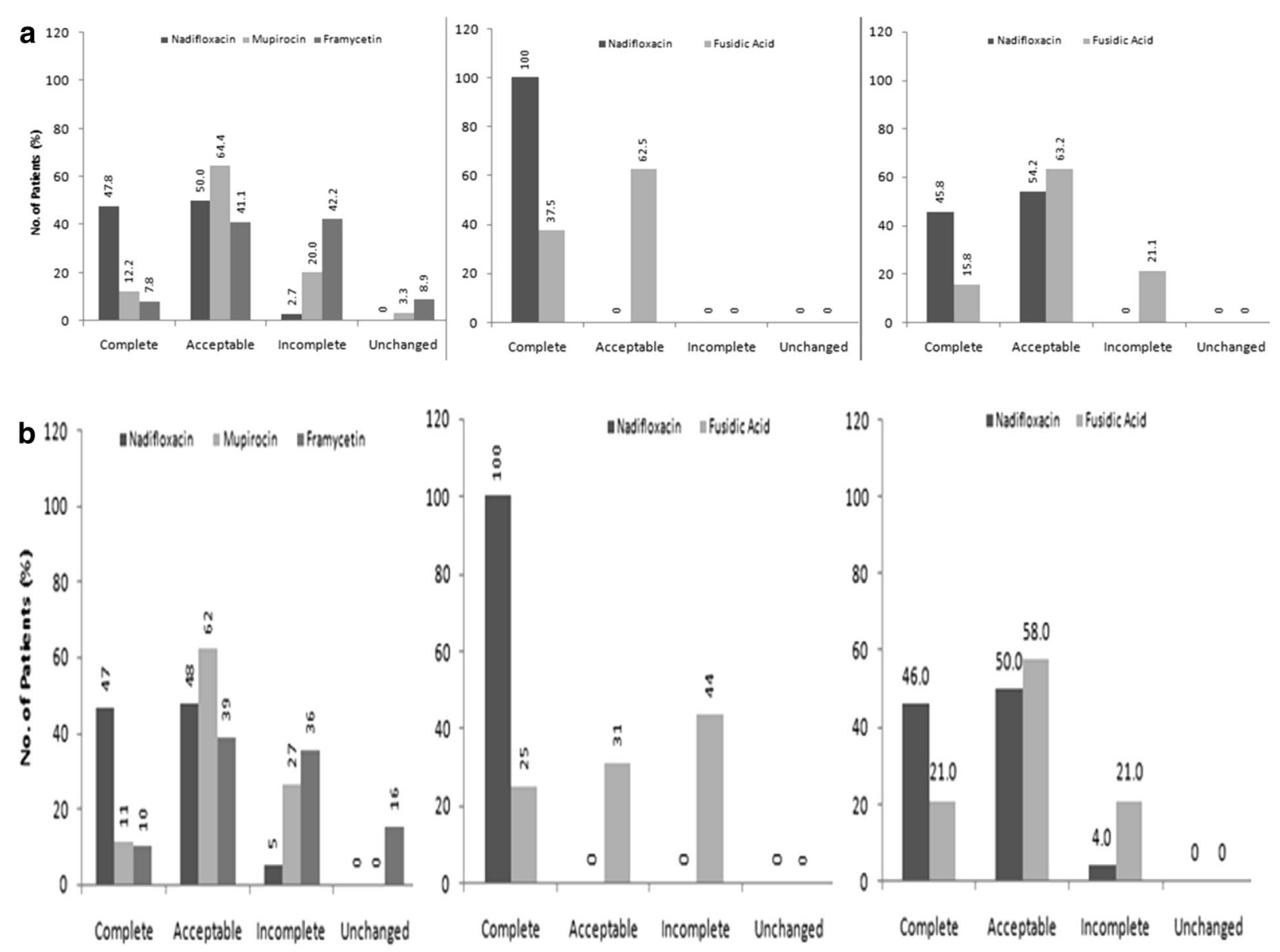

C

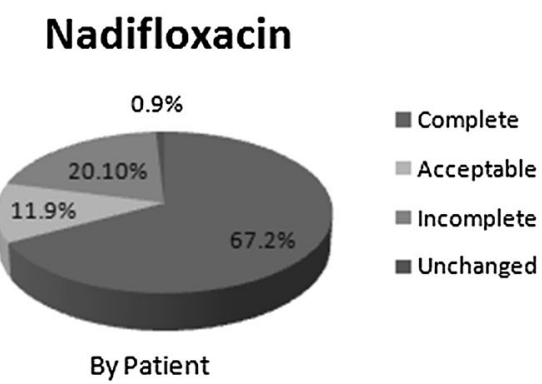

Fig. 4 a Global assessment of nadifloxacin and other comparator drugs by patients in study 1 , study 2 and study 3. b Global assessment of patient by investigator in study 1 ,

\section{Secondary Parameters}

Global Assessment by Patients The efficacy of nadifloxacin in treatment of bacterial skin infections was rated by patients as excellent (complete remission of symptoms) for 221

\section{Nadifloxacin}

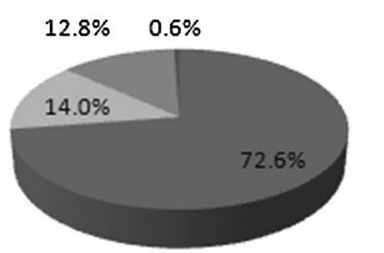

By Investigator
- Complete

acceptable

Incomplete

unchanged study 2 and study 3 treated with nadifloxacin and comparator drugs. c Global assessments by patients (left) and investigator (right) in the post-marketing surveillance

(67.2\%) patients, good (acceptable remission of symptoms) in 39 (11.9\%) patients, fair (slight/incomplete remission of symptoms) in $66(20.1 \%)$ patients and poor (unchanged/ aggravated) in 3 (0.9\%) patients (Fig. 4c). 
Table 2 Evaluation of symptoms before and after the treatment of bacterial skin infections with nadifloxacin

\begin{tabular}{|c|c|c|c|c|c|c|c|c|c|}
\hline \multirow[t]{2}{*}{ Parameter } & \multicolumn{3}{|l|}{ Pre-study } & \multicolumn{3}{|l|}{ Day 7} & \multicolumn{3}{|l|}{ Day 14} \\
\hline & $\begin{array}{l}\text { Mild } \\
n(\%)\end{array}$ & $\begin{array}{l}\text { Moderate } \\
n(\%)\end{array}$ & $\begin{array}{l}\text { Severe } \\
n(\%)\end{array}$ & $\begin{array}{l}\text { Mild } \\
n(\%)\end{array}$ & $\begin{array}{l}\text { Moderate } \\
n(\%)\end{array}$ & $\begin{array}{l}\text { Severe } \\
n(\%)\end{array}$ & $\begin{array}{l}\text { Mild } \\
n(\%)\end{array}$ & $\begin{array}{l}\text { Moderate } \\
n(\%)\end{array}$ & $\begin{array}{l}\text { Severe } \\
n(\%)\end{array}$ \\
\hline Erythema & $87(26.4)$ & $114(34.7)$ & $27(8.2)$ & $101(30.7)$ & $9(2.7)$ & $0(0.0)$ & $12(3.6)$ & $1(0.3)$ & $0(0.0)$ \\
\hline Exudation & $73(22.2)$ & $66(20.1)$ & $17(5.2)$ & $56(17.0)$ & $12(3.6)$ & $0(0.0)$ & $5(1.5)$ & $2(0.6)$ & $0(0.0)$ \\
\hline Swelling & $81(24.6)$ & $81(24.6)$ & $20(6.1)$ & $66(20.1)$ & $14(4.3)$ & $0(0.0)$ & $10(3.0)$ & $2(0.6)$ & $0(0.0)$ \\
\hline Pruritus & $66(20.1)$ & $43(13.1)$ & $29(8.8)$ & $58(17.6)$ & $7(2.1)$ & $1(0.3)$ & $8(2.4)$ & $0(0.0)$ & $0(0.0)$ \\
\hline Crusting & $68(20.7)$ & $65(19.8)$ & $8(2.4)$ & $45(13.7)$ & $9(2.7)$ & $1(0.3)$ & $9(2.7)$ & $1(0.3)$ & $0(0.0)$ \\
\hline Pain & $93(28.3)$ & $87(26.4)$ & $28(8.5)$ & $50(15.2)$ & $13(4.0)$ & $2(0.6)$ & $8(2.4)$ & $1(0.3)$ & $0(0.0)$ \\
\hline Tenderness & $75(22.8)$ & $74(22.5)$ & $24(7.3)$ & $48(14.6)$ & $7(2.1)$ & $1(0.3)$ & $5(1.5)$ & $1(0.3)$ & $0(0.0)$ \\
\hline
\end{tabular}

$N$ number of patients

\section{Global Assessment by Investigator The} efficacy of nadifloxacin in treatment of bacterial skin infections was evaluated by investigator on a 4-point scale as excellent (complete remission of symptoms) for 239 (72.6\%) patients, good (acceptable remission of symptoms) in $46(14.0 \%)$ patients, fair (slight/incomplete remission of symptoms) in $42(12.8 \%)$ patients and poor (unchanged/ aggravated) in 2 (0.6\%) patients (Fig. $4 \mathrm{c}$ ).

\section{Safety Evaluation}

Safety was assessed based on AEs following the treatment. Study 1, 2 and 3 did not report any $\mathrm{AE}$ as in the phase IV study, two AEs (burning and itching) were reported, one in each patient. Of the two AEs, itching persisted whereas the burning improved at the time of reporting. Of the two AEs, the causal relationship of the $\mathrm{AE}$ in one patient was "Definitely Related" whereas in the other patient the causal relationship of the $\mathrm{AE}$ was "Unrelated", as per the investigator/prescriber's assessment. No serious AEs were reported in the studies.

\section{DISCUSSION}

The results of all the three comparative, randomized clinical studies and the PMS showed that nadifloxacin is well tolerated and efficacious in the treatment of patients with bacterial skin infections. There was a significant reduction in mean severity scores for all the symptoms of bacterial infections (including erythema, crusting, exudation, swelling, pruritus, pain, and tenderness) in the nadifloxacin groups of all the three clinical studies as compared with other study groups. In all the studies (clinical and PMS), nadifloxacin was rated as excellent (complete remission of symptoms) on a 4-point scale by a significantly higher number of patients as compared with mupirocin, framycetin, and fusidic acid. Investigators also rated the overall response to the application of the study cream on a 4-point scale as excellent (complete remission of symptoms) in significantly higher number of patients in the nadifloxacin groups. Minimal AEs were reported.

Several antibiotics (fusidic acid, mupirocin, framycetin and fluoroquinolones including 
ciprofloxacin, levofloxacin, gemifloxacin, and moxifloxacin) are available for the treatment of bacterial infections $[3-5,19,20]$. Nadifloxacin is a new chemically synthesized fluoroquinolone that has C-6 substituted with flouro group. The presence of fluoro group enhances the antibacterial activity of nadifloxacin probably by improving its ability to bind the DNA gyrase complex (2- to 17-fold) and cell penetration (1to 70 -fold) as compared to quinolones with no substitution [21, 22]. In vitro and clinical studies have already proven the safety and efficacy of nadifloxacin in the treatment of acne vulgaris and suggested that nadifloxacin could be used as an effective therapeutic agent in treatment of other bacterial infections [14$16,23,24]$. All the studies conducted to date (both in vitro and in vivo) have shown nadifloxacin to be a superior treatment for bacterial skin infections. Nadifloxacin has also been reported to be effective against bacteria, like MRSA, that have developed resistance to other available anti-microbial [9, 13, 25].

Previous in vitro studies have assessed the activity of nadifloxacin against bacterial skin infection causing organisms. Nenoff et al. [10] conducted an in vitro study that compared and assessed the activity of nadifloxacin with various other anti-bacterials including oxacillin, flucloxacillin, ofloxacin, erythromycin, cefotiam, clindamycin and gentamicin against aerobic and anaerobic Gram-positive bacteria including $S$. aureus, coagulase-negative Staphylococci (CNS), Streptococcus spp., Propionibacterium granulosum, Propionibacterium acnes strains. The results demonstrated nadifloxacin to be highly active against all bacteria except some of the CNS strains. Minimum inhibitory concentration (MIC $_{90}$ at which $90 \%$ of bacteria are inhibited) of nadifloxacin was $0.1 \mu \mathrm{g} / \mathrm{mL}$ for $S$. aureus, $0.39 \mu \mathrm{g} / \mathrm{mL}$ for Propionibacterium spp., and
$0.78 \mu \mathrm{g} / \mathrm{mL}$ for both Streptococcus spp. and CNS, which was significantly less than MICs for other test antibiotics. No resistant strains to nadifloxacin were found in this study except for some CNS strains in agreement with the study by Vogt et al, who also reported the lowest incidence of resistance of nadifloxacin against the strains of CNS, $S$. aureus, $P$. acnes, and $P$. granulosum [10, 26].

Another in vitro study, which compared the activity of nadifloxacin with ciprofloxacin, erythromycin and clindamycin against isolates of $P$. acnes, $S$. epidermidis, and both methicillinsusceptible and -resistant $S$. aureus (MSSA and MRSA, respectively) taken from Spain, Hungary and Germany showed that nadifloxacin possesses better activity against strains of $P$. acnes than other test antibiotics as the $\mathrm{MIC}_{50}$ (at which $50 \%$ of bacteria are inhibited) and $\mathrm{MIC}_{90}$ values (range between 0.03 and $1 \mathrm{mg} / \mathrm{L}$ ) for nadifloxacin were lesser than $\mathrm{MIC}_{50}$ and $\mathrm{MIC}_{90}$ values of other test antibiotics. The study observed that MSSA and MRSA strains were more resistant to ciprofloxacin as compared to nadifloxacin with exception of the MSSA isolates from Spain in which $7.5 \%$ were resistant to nadifloxacin [13]. Jacobs and associates assessed the in vitro activity of nadifloxacin against quinolone-susceptible and -resistant S. aureus and S. epidermidis. The MIC of nadifloxacin was less than all the other currently available quinolones (clinafloxacin, moxifloxacin, vancomycin, levofloxacin, trovafloxacin, ciprofloxacin, teicoplanin) which showed it to be the most potent therapeutic agent against the tested Staphylococci spp. (both quinolones susceptible and resistant) [12].

There are limited clinical studies conducted to assess the safety and efficacy of nadifloxacin in treatment of bacterial skin infections. Haustein and colleagues investigated the 
efficacy and tolerability of nadifloxacin in treatment of patients with bacterial infections including impetigo contagiosa, folliculitis/ sycosis vulgaris, impetiginized dermatitis and secondarily infected wounds. Statistically significant reduction in the degree of infections was observed and physicians rated the therapeutic effect of nadifloxacin on a 4point scale as 'very good' in more patients similar to that observed in our studies [17].

Resistance to anti-microbials is a menace in treating bacterial infections. Drug export by efflux pumps is a common mechanism for antimicrobial drug resistance. Bacteria encode several efflux pumps; extensive research is reported on the NorA efflux pump. NorA efflux pump is used by bacteria (such as $S$. aureus) to pump out drugs leading to drug resistance [27]. This inhibits the activity of drugs on bacteria. Fluoroquinolones such as ciprofloxacin, levofloxacin, and moxifloxacin have shown resistance to both Gram-positive (including Staphylococcus species) and Gramnegative bacteria. However, nadifloxacin is not influenced by over expression of the NorA efflux pump on the bacterial cell membrane which reduces the chances of development of resistance [28, 29]. A previous study determined that nadifloxacin acts independent of NorA efflux pump, as MIC for $\mathrm{NorA}^{+} S$. aureus was only one fold higher than that of NorA ${ }^{-}$ strain [12]. Several other studies demonstrated nadifloxacin to have negligible or low resistance against strains of $S$. aureus $[10,26,30]$ and good susceptibility to all bacteria that are resistant to fluoroquinolones currently in use [10, 31, 32].

The authors' studies were the first to compare nadifloxacin with fusidic acid, framycetin, and mupirocin for the treatment of bacterial skin infection. The use of fusidic acid, framycetin, and mupirocin in the treatment of bacterial infections has been investigated in several studies. A study, by Gisby and co-workers, in mouse models with skin wound infections compared the efficacy of mupirocin with other antibiotics including fusidic acid, neomycin-bacitracin, erythromycin, cephalexin, benzylpenicillin, methicillin and flucloxacillin used in the treatment of primary and secondary bacterial infections. In vitro results found that MIC value of mupirocin for $S$. aureus was $0.12 \mu \mathrm{g} / \mathrm{mL}$, which is more than that reported by Nenoff et al. in which the MIC value of nadifloxacin was $0.1 \mu \mathrm{g} / \mathrm{mL}$ for $S$. aureus. This indicates that nadifloxacin is a better option than mupirocin and other comparator antibiotics used in this study [4]. Another randomized clinical trial reported that fusidic acid resistant strains of MRSA were found in isolates of patients treated with fusidic acid [33].

Spelman and his colleagues gathered in vitro and clinical studies data to assess the efficacy and safety of fusidic acid in treatment of patients with bacterial infections. Though the study demonstrated that fusidic acid is effective against Staphylococci, Streptococci were resistant to fusidic acid. However, nadifloxacin has shown good efficacy against both Staphylococci and Streptococci [10, 11].

Anti-bacterials currently used in the treatment of bacterial skin infections such as fusidic acid, mupirocin, and quinolones including ciprofloxacin, levofloxacin, moxifloxacin and gemifloxacin have been reported to cause several AEs [14, 34-37]. However, nadifloxacin, a synthetic quinolone, has been reported to cause minimal/negligible AEs in the treatment of acne vulgaris. The data on its AEs for use in the treatment of bacterial skin infections are limited. Haustein et al. reported AEs including itching, erythema and inflammatory swelling in only three patients [7, $14,17,38]$. The authors did not observe any AE 
in these clinical studies and the phase IV study found only two AEs (burning and itching) and of the two AEs, burning, improved at the time of reporting. No serious AEs were reported in the studies.

\section{CONCLUSION}

In the present study, the authors have reported the results of three randomized clinical studies and a PMS on the use of nadifloxacin in treatment of bacterial skin infections in Indian patients. They compared nadifloxacin with other anti-bacterials used for treatment of bacterial skin infections and showed nadifloxacin to be safe and superior treatment for bacterial skin diseases.

Future clinical trials of nadifloxacin in the treatment of bacterial skin infections should be conducted to assess long-term efficacy and safety.

\section{ACKNOWLEDGMENTS}

All named authors meet the ICMJE criteria for authorship for this manuscript, take responsibility for the integrity of the work as a whole, and have given final approval for the version to be published. Sponsorship and article processing charges for this study was funded by Wockhardt Ltd. (Mumbai, India). Medical writing assistance for this study was provided by Anupam Sharma (Knowledge Isotopes Pvt. Ltd., Mohali, India) and was funded by Wockhardt Ltd. (Mumbai, India).

Conflict of interest. S Motlekar is an employee of Wockhardt Ltd. (Mumbai, India). V Narayanan is an employee of Wockhardt Ltd. (Mumbai, India). G Kadhe is an employee of Wockhardt Ltd. (Mumbai, India). S Bhagat is an employee of Wockhardt Ltd. (Mumbai, India).

Compliance with ethics guidelines. All procedures followed were in accordance with the ethical standards of the responsible committee on human experimentation (institutional and national) and with the Helsinki Declaration of 1975 , as revised in 2000 and 2008. Informed consent was obtained from all patients for being included in the studies.

Funding. Sponsorship and article processing charges for this study was funded by Wockhardt Ltd (Mumbai, India).

Open Access. This article is distributed under the terms of the Creative Commons Attribution Noncommercial License which permits any noncommercial use, distribution, and reproduction in any medium, provided the original author(s) and the source are credited.

\section{REFERENCES}

1. Ki V, Rotstein C. Bacterial skin and soft tissue infections in adults: a review of their epidemiology, pathogenesis, diagnosis, treatment and site of care. Can J Infect Dis Med Microbiol. 2008;19(2):173-84.

2. Indian Network for Surveillance of Antimicrobial Resistance (INSAR) group. Methicillin resistant Staphylococcus aureus (MRSA) in India: prevalence $\&$ susceptibility pattern. Indian J Med Res. 2013;137(2):363-9.

3. Hetem DJ, Bonten MJ. Clinical relevance of mupirocin resistance in Staphylococcus aureus. J Hosp Infect. 2013;85(4):249-56.

4. Gisby J, Bryant J. Efficacy of a new cream formulation of mupirocin: comparison with oral and topical agents in experimental skin infections. Antimicrob Agents Chemother. 2000;44(2):255-60.

5. Spelman D. Fusidic acid in skin and soft tissue infections. Int J Antimicrob Agents. 1999;12(Suppl 2):S59-66. 
6. Takei M, et al. Target preference of 15 quinolones against Staphylococcus aureus, based on antibacterial activities and target inhibition. Antimicrob Agents Chemother. 2001;45(12):3544-7.

7. Plewig G, Holland KT, Nenoff P. Clinical and bacteriological evaluation of nadifloxacin $1 \%$ cream in patients with acne vulgaris: a doubleblind, phase III comparison study versus erythromycin 2\% cream. Eur J Dermatol. 2006;16(1):48-55.

8. de Souza NJ, et al. A chiral benzoquinolizine-2carboxylic acid arginine salt active against vancomycin-resistant Staphylococcus aureus. J Med Chem. 2005;48(16):5232-42.

9. Patel MV, et al. Antistaphylococcal activity of WCK 771, a tricyclic fluoroquinolone, in animal infection models. Antimicrob Agents Chemother. 2004;48(12):4754-61.

10. Nenoff P, Haustein UF, Hittel N. Activity of nadifloxacin (OPC-7251) and seven other antimicrobial agents against aerobic and anaerobic Gram-positive bacteria isolated from bacterial skin infections. Chemotherapy. 2004;50(4):196-201.

11. Jacobs MR, Appelbaum PC. Nadifloxacin: a quinolone for topical treatment of skin infections and potential for systemic use of its active isomer, WCK 771. Expert Opin Pharmacother. 2006;7(14):1957-66.

12. Jacobs MR, et al. In vitro activity of the new quinolone WCK 771 against staphylococci. Antimicrob Agents Chemother. 2004;48(9):3338-42.

13. Alba $\mathrm{V}$, et al. In vitro activity of nadifloxacin against several Gram-positive bacteria and analysis of the possible evolution of resistance after 2 years of use in Germany. Int $\mathrm{J}$ Antimicrob Agents. 2009;33(3):272-5.

14. Choudhury S, et al. Efficacy and safety of topical nadifloxacin and benzoyl peroxide versus clindamycin and benzoyl peroxide in acne vulgaris: a randomized controlled trial. Indian J Pharmacol. 2011;43(6):628-31.

15. Schöfer H, Göllner A, Kusche W, Schwantes U. Effectiveness and tolerance of topical nadifloxacin in the therapy of acne vulgaris (grade I-II): results of a non-interventional trial in 555 patients. J Appl Res. 2009;9:44-51.

16. Kobayashi M, et al. Efficacy of combined topical treatment of acne vulgaris with adapalene and nadifloxacin: a randomized study. J Dermatol. 2011;38(12):1163-6.
17. Haustein UF, Nenoff P, Hittel N. Topical quinolone nadifloxacin (OPC-7251) in bacterial skin disease: clinical evaluation in a multicenter open trial and in vitro anti microbiological susceptibility testing. J Dermatol Treat. 1997;8:87-92.

18. Jones RN. Fluoroquinolone resistance. An evolving national problem or just a problem for some physicians? Diagn Microbiol Infect Dis. 1992;15(2):177-9.

19. Luke RG, Watson WC. Antibiotics in dermatology. Br Med J. 1963;2(5363):981-2.

20. Blondeau JM. The role of fluoroquinolones in skin and skin structure infections. Am J Clin Dermatol. 2002;3(1):37-46.

21. Koga $\mathrm{H}$, et al. Structure-activity relationships of antibacterial 6,7- and 7,8-disubstituted 1-alkyl-1,4dihydro-4-oxoquinoline-3-carboxylic acids. J Med Chem. 1980;23(12):1358-63.

22. Chu DT, Fernandes PB. Structure-activity relationships of the fluoroquinolones. Antimicrob Agents Chemother. 1989;33(2):131-5.

23. Gollnick HPM, Vogt K, Hermann J, et al. Topical quinolone OPC-7251: a clinical and microbiological study in acne. Eur J Dermatol. 1994;4:210-15.

24. Kurokawa I, et al. Clinical and bacteriologic evaluation of OPC-7251 in patients with acne: a double-blind group comparison study versus cream base. J Am Acad Dermatol. 1991;25(4):674-81.

25. Kimata H. Effect of nadifloxacin on atopic dermatitis with methicillin-resistant Staphylococcus aureus in young children. Eur J Pediatr. 1999;158(11):949.

26. Vogt $\mathrm{K}$, et al. Comparative activity of the topical quinolone OPC-7251 against bacteria associated with acne vulgaris. Eur J Clin Microbiol Infect Dis. 1992;11(10):943-5.

27. Deng $X$, et al. Expression of multidrug resistance efflux pump gene norA is iron responsive in Staphylococcus aureus. J Bacteriol. 2012;194(7):1753-62.

28. Morrow BJ, et al. Antistaphylococcal activities of the new fluoroquinolone JNJ-Q2. Antimicrob Agents Chemother. 2011;55(12):5512-21.

29. Guilhelmelli F, et al. Antibiotic development challenges: the various mechanisms of action of antimicrobial peptides and of bacterial resistance. Front Microbiol. 2013;4:353. 
30. Oizumi N, et al. Relationship between mutations in the DNA gyrase and topoisomerase IV genes and nadifloxacin resistance in clinically isolated quinolone-resistant Staphylococcus aureus. J Infect Chemother. 2001;7(3):191-4.

31. Madhusudhan KT, et al. Comparative in vitro activity of three fluoroquinolones against clinical isolates by $E$ test. Chemotherapy. 2003;49(4):184-8.

32. Raviglione MC, et al. Ciprofloxacin-resistant methicillin-resistant Staphylococcus aureus in an acute-care hospital. Antimicrob Agents Chemother. 1990;34(11):2050-4.

33. Chang SC, et al. Oral fusidic acid fails to eradicate methicillin-resistant Staphylococcus aureus colonization and results in emergence of fusidic acid-resistant strains. Diagn Microbiol Infect Dis. 2000;36(2):131-6.

34. Rist $\mathrm{T}$, et al. A comparison of the efficacy and safety of mupirocin cream and cephalexin in the treatment of secondarily infected eczema. Clin Exp Dermatol. 2002;27(1):14-20.

35. Stahlmann R, Lode H. Safety considerations of fluoroquinolones in the elderly: an update. Drugs Aging. 2010;27(3):193-209.

36. Iannini PB. The safety profile of moxifloxacin and other fluoroquinolones in special patient populations. Curr Med Res Opin. 2007;23(6):1403-13.

37. Liu HH. Safety profile of the fluoroquinolones: focus on levofloxacin. Drug Saf. 2010;33(5):353-69.

38. Jung JY, et al. Clinical and histological evaluation of $1 \%$ nadifloxacin cream in the treatment of acne vulgaris in Korean patients. Int $\mathrm{J}$ Dermatol. 2011;50(3):350-7. 